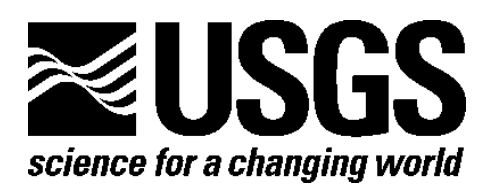

\title{
Seasonal Flux and Assemblage Composition of Planktic Foraminifera from the Northern Gulf of Mexico, 2008-11
}

By Caitlin E. Reynolds and Richard Z. Poore

Open-File Report 2013-1158

U.S. Department of the Interior

U.S. Geological Survey 


\section{U.S. Department of the Interior \\ SALLY JEWELL, Secretary}

\section{U.S. Geological Survey \\ Suzette M. Kimball, Acting Director}

U.S. Geological Survey, Reston, Virginia: 2013

For more information on the USGS-the Federal source for science about the Earth, its natural and living resources, natural hazards, and the environment-visit http://www.usgs.gov or call 1-888-ASK-USGS

For an overview of USGS information products, including maps, imagery, and publications, visit $h$ ttp://www.usgs.gov/pubprod

To order this and other USGS information products, visit $h$ ttp://store.usgs.gov

Suggested citation:

Reynolds, C.E., and Poore, R.Z., 2013, Seasonal flux and assemblage composition of planktic foraminifera from the northern Gulf of Mexico, 2008-11: U.S. Geological Survey Open-File Report 2013-1158, 11 p.

Any use of trade, product, or firm names is for descriptive purposes only and does not imply endorsement by the U.S. Government.

Although this report is in the public domain, permission must be secured from the individual copyright owners to reproduce any copyrighted material contained within this report. 


\section{Contents}

Abstract
Introduction
Regional Setting
Materials and Methods
Results from 2011
Discussion
Conclusions
Acknowledgments
References Cited

\section{Figures}

1. Location of the sediment-trap mooring (inverted triangle) in the northern Gulf of Mexico at approximately $25.7^{\circ} \mathrm{N}$ latitude and $90.3^{\circ} \mathrm{W}$ longitude.

2. Temperature depth profiles of World Ocean Atlas 2009 climatology (monthly average) (WOA09) (black lines) and conductivity-temperature-depth (CTD) casts for the sediment trap during 2008 (blue lines), 2009 (red lines), 2010 (green lines), and 2011 (brown lines).

3. Salinity depth profiles of World Ocean Atlas 2009 climatology (monthly average) (WOA09) (black lines) and conductivity-temperature-depth (CTD) casts for the sediment trap during 2008 (blue lines), 2009 (red lines), 2010 (green lines), and 2011 (brown lines).

4. Average daily flux of 7- to 14-day-long sampling intervals of all planktic foraminifers and the 10 most abundant species/groups during 2008-11. Note the scale change in the $y$-axis. The tick marks on the $x$-axis denote the $1^{\text {st }}$ day of each month. There was a gap in sampling from late May to late September 2009 (red rectangles) and loss of nine sample cups (red bars). $\mathrm{m}^{-2}$ day $^{-1}$, square meter per day......

5. Weekly percent abundance of the 10 most common species/groups of planktic foraminifers during 2008-11. Note the scale change in the $y$-axis. The tick marks on the $x$-axis denote the 1 st day of each month. There was a gap in sampling from late May to late September 2009 (red rectangles) and loss of nine sample cups (red bars).

\section{Tables}

1. Planktic foraminiferal flux and percent contribution to the total assemblage for the 14 most common species, northern Gulf of Mexico 


\title{
Seasonal Flux and Assemblage Composition of Planktic Foraminifera from the Northern Gulf of Mexico, 2008-11
}

\author{
By Caitlin E. Reynolds and Richard Z. Poore
}

\begin{abstract}
The U.S. Geological Survey anchored a sediment trap in the northern Gulf of Mexico to collect seasonal time-series data on the flux and assemblage composition of live planktic foraminifers. This report provides an update of the previous time-series data to include results from 2011. Ten species, or varieties, constituted $~ 92$ percent of the 2011 assemblage: Globigerinoides ruber (pink and white varieties), Globigerinoides sacculifer, Globigerina calida, Globigerinella aequilateralis, Globorotalia menardii group [The Gt. menardii group includes Gt. menardii, Gt. tumida, and Gt. ungulata], Orbulina universa, Globorotalia truncatulinoides, Pulleniatina spp., and Neogloboquadrina dutertrei. The mean daily flux was 205 tests per square meter per day $\left(\mathrm{m}^{-2}\right.$ day $\left.{ }^{-1}\right)$, with maximum fluxes of $>600$ tests $\mathrm{m}^{-2}$ day $^{-1}$ during mid-February and mid-September and minimum fluxes of $<60$ tests $\mathrm{m}^{-2}$ day $^{-1}$ during mid- $^{-}$ March, the beginning of May, and November. Globorotalia truncatulinoides showed a clear preference for the winter, consistent with data from 2008 to 2010. Globigerinoides ruber (white) flux data for 2011 (average 30 tests $\mathrm{m}^{-2}$ day $^{-1}$ ) were consistent with data from 2010 (average $29 \mathrm{~m}^{-2}$ day $^{-1}$ ) and showed a steady threefold increase since 2009 (average 11 tests $\mathrm{m}^{-2}$ day $^{-1}$ ) and a tenfold increase from the 2008 flux $\left(3\right.$ tests $\mathrm{m}^{-2}$ day $\left.^{-1}\right)$.
\end{abstract}

\section{Introduction}

A sediment trap was moored in the northern Gulf of Mexico in January 2008 as part of a U.S. Geological Survey Mendenhall Postdoctoral Fellowship project. The sediment trap, equipped with an automated sampling system, has continuously collected material in the water column from January 2008 to the present and is currently deployed. Information on the trap, trap mooring, planktic foraminifers as climate proxies, and the results from the first year are detailed in Tedesco and others (2009). In this report, we update results from the sediment-trap series to include material collected between January and December of 2011. The report presents the data without interpretation. For discussion and interpretation of flux data from 2008 to 2010, see Poore and others, 2013.

\section{Regional Setting}

The Gulf of Mexico is a semi-enclosed basin surrounded by the Gulf Coast of the United States, Mexico, and Cuba (fig. 1). Sea-surface temperature (SST) at the trap site ranges from a winter low of approximately 21 degrees Celsius $\left({ }^{\circ} \mathrm{C}\right.$ ) to a high of $30{ }^{\circ} \mathrm{C}$ (World Ocean Atlas 2009 data cited in Locarnini and others, 2010). Sea-surface salinity (SSS) ranges from about 35 practical salinity units (psu) in the winter to $32 \mathrm{psu}$ in the summer (World Ocean Atlas 2009 data cited in Antonov and others, 2010). 
The Gulf of Mexico is connected to the Caribbean and tropical North Atlantic through the Loop Current. The Loop Current is a surface current that enters the Gulf of Mexico between Cuba and the Yucatan Peninsula and typically loops east and south before exiting through the Florida Straits.

The Gulf of Mexico, Caribbean Sea, and western tropical North Atlantic compose the Atlantic Warm Pool, the Atlantic portion of the Western Hemisphere Warm Pool (Wang and Enfield, 2001). The Atlantic Warm Pool is defined by the region covered by water warmer than $28.5^{\circ} \mathrm{C}$ and constitutes a large part of the tropical heat engine, supplying moisture to the atmosphere and latent heat to North America from early spring to early fall (Wang and Enfield, 2001; Wang and others, 2006). World Ocean Atlas 2009 climatology indicates the trap site is part of the Atlantic Warm Pool during July, August, and September (summer) (Locarnini and others, 2010).

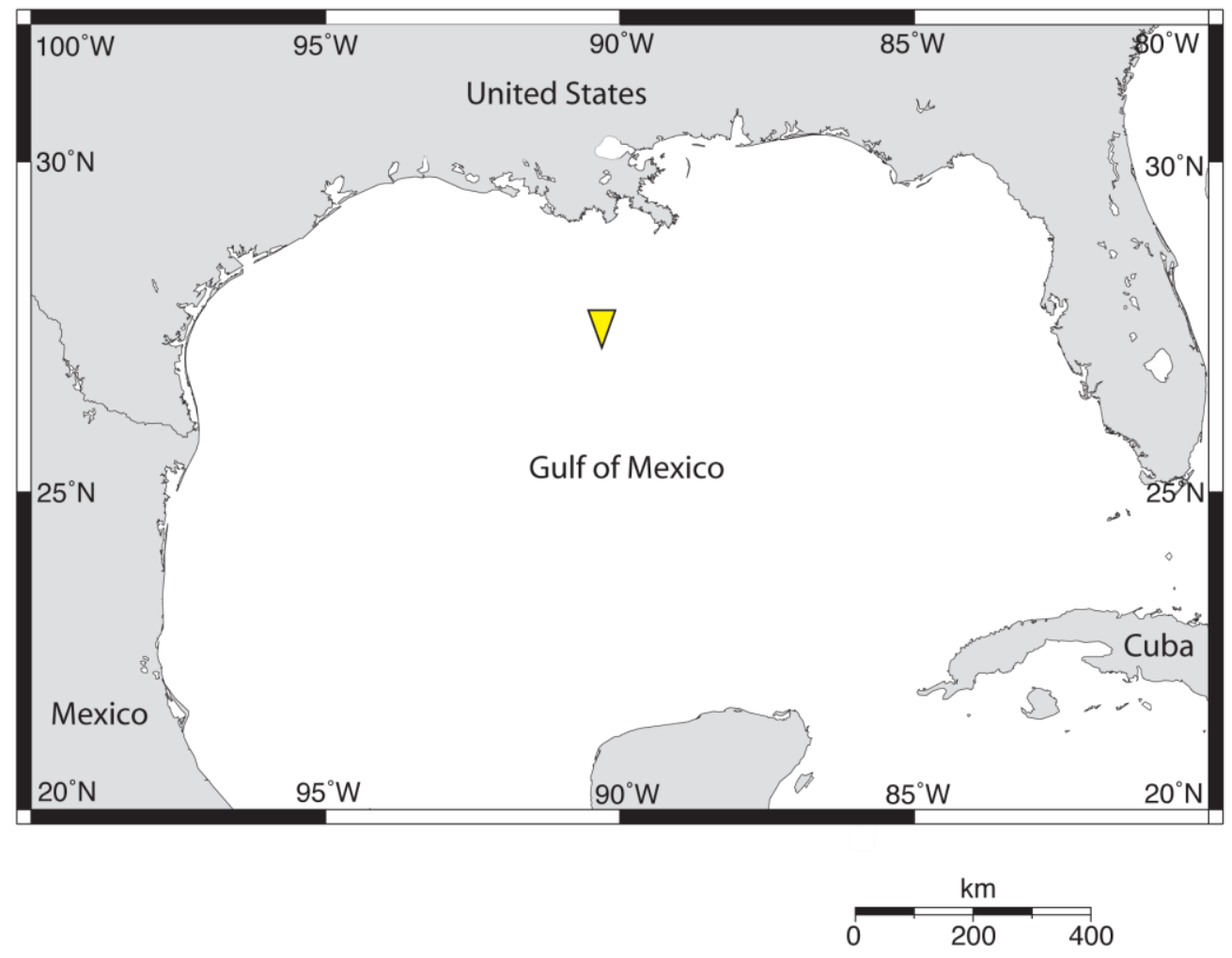

Figure 1. Location of the sediment-trap mooring (inverted triangle) in the northern Gulf of Mexico at approximately $25.7^{\circ} \mathrm{N}$ latitude and $90.3^{\circ} \mathrm{W}$ longitude.

\section{Materials and Methods}

A McLane PARFLUX Mark 78 automated sediment trap was deployed in early January 2008 in approximately 1,150 meters $(\mathrm{m})$ of water depth at approximately $27.5^{\circ} \mathrm{N}$ latitude and $90.3^{\circ} \mathrm{W}$ longitude. The trap is positioned at a depth of $700 \mathrm{~m}$ on the mooring to guarantee the collection of deeper-dwelling species of planktic foraminifers (for example, Globorotalia spp.). The trap is equipped with 21 collection cups that are mounted on a rotating plate that is programmed to rotate every 7 to 14 days (representing a 1- or 2-week collection period). Sample cups from January 2008 to late May 2009 contained a buffered formalin solution made with filtered $(0.44$-micrometer $(\mu \mathrm{m})$ filter) seawater, with a salinity of approximently 33 psu. Sample cups for September 2009 to the present contain a density- 
gradient solution with a salinity of approximately 44 psu. Formalin (3.7 percent) and sodium borate are added to the density-gradient solution to poison and preserve the samples. The trap was recovered and redeployed every 3 months during 2008 and approximately every 6 months thereafter. A gap in our sampling occurred from late May to late September 2009 due to scheduling problems. Nine samples from the weeks of March 17, April 7, May 5, October 22, November 19, and December 10 of 2009, and January 7, February 14 and 24 of 2010 were not recovered due to loss of the cups during deployment/recovery. During visits to the trap site, conductivity-temperature-depth (CTD) measurements were collected using a Sea-Bird Electronics SBE9plus (figs. 2 and 3).

Temperature $\left({ }^{\circ} \mathrm{C}\right)$
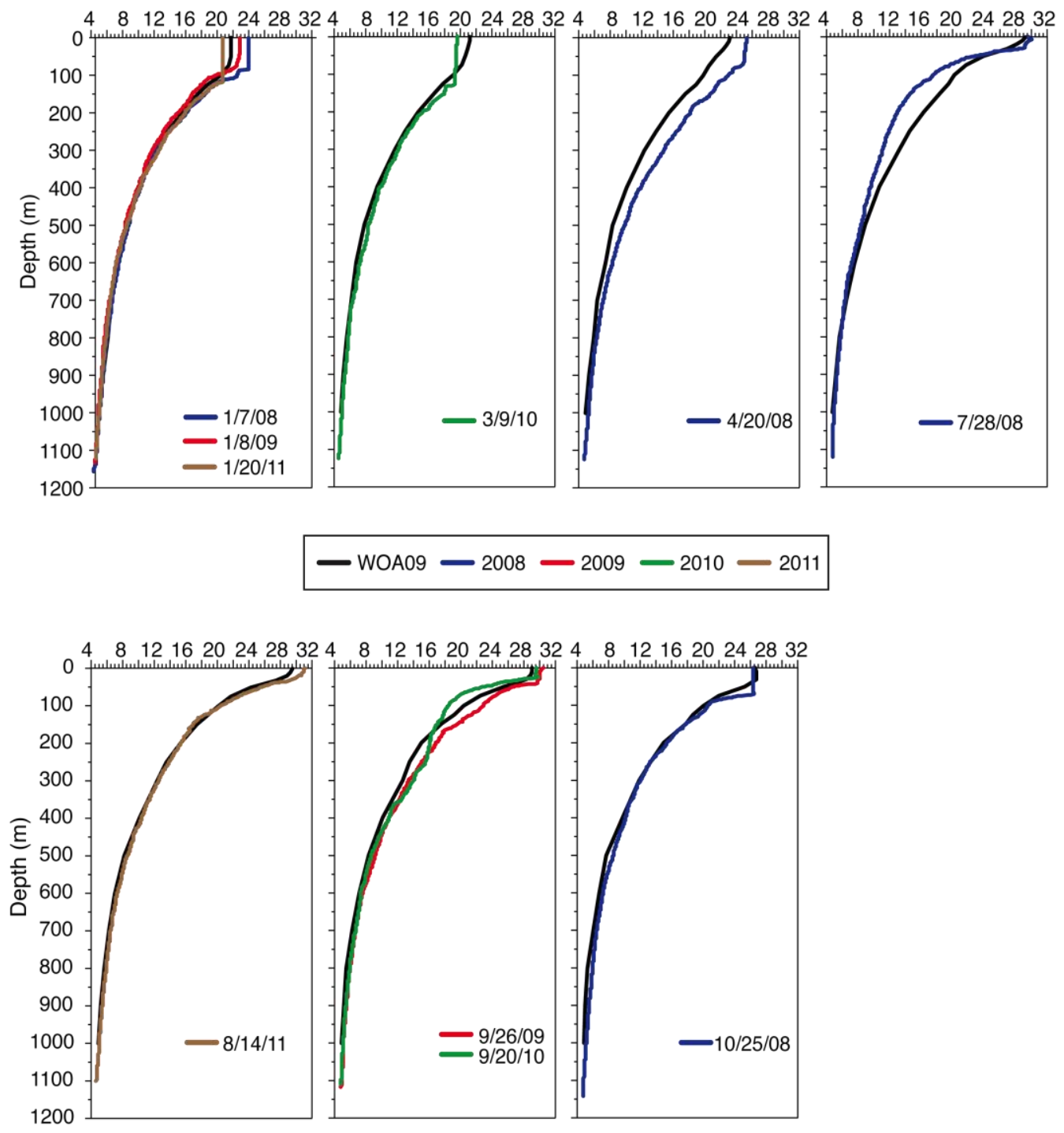

Figure 2. Temperature depth profiles of World Ocean Atlas 2009 climatology (monthly average) (WOA09) (black lines) and conductivity-temperature-depth (CTD) casts for the sediment trap during 2008 (blue lines), 2009 (red lines), 2010 (green lines), and 2011 (brown lines). 
Salinity (psu)
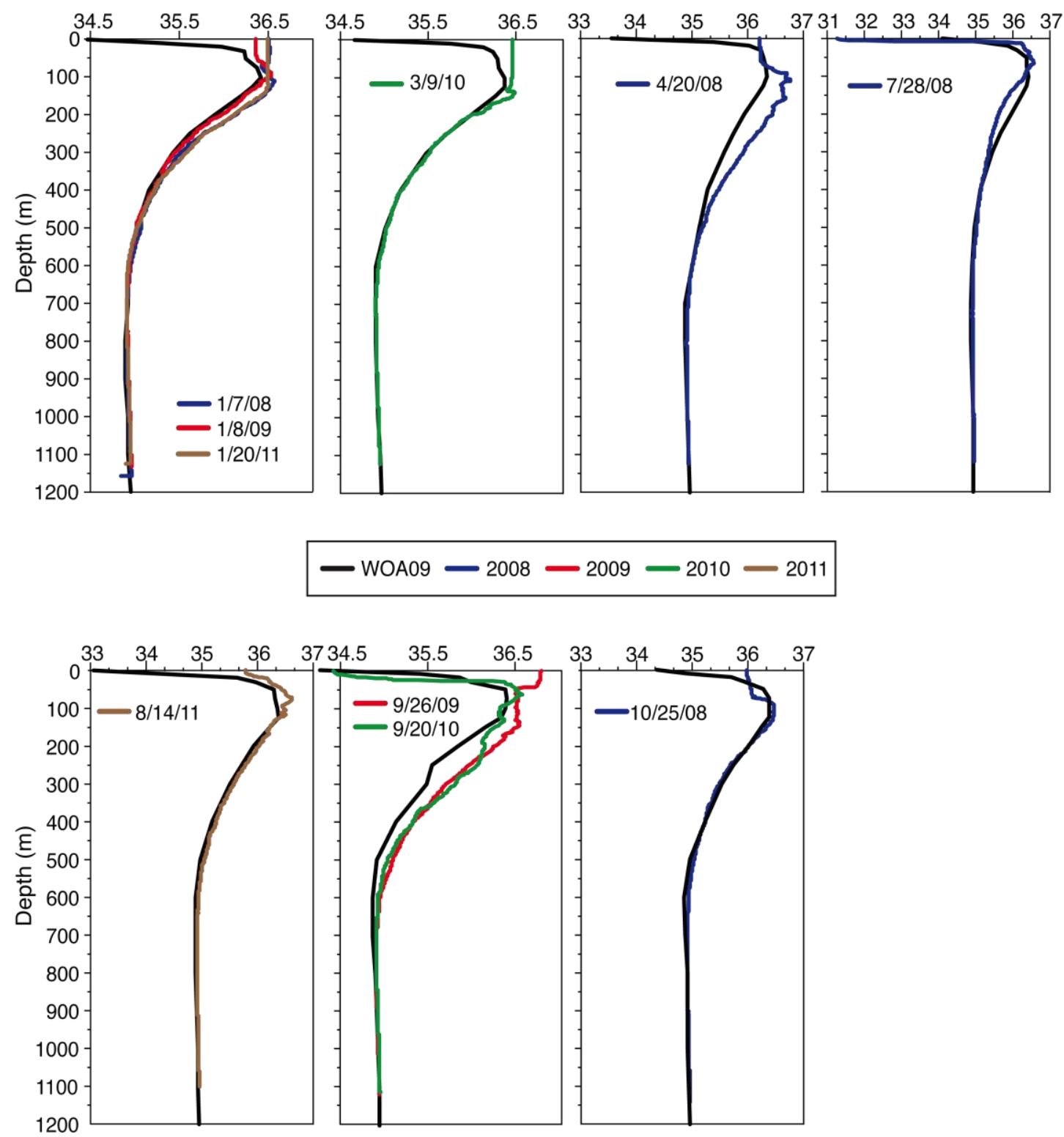

Figure 3. Salinity depth profiles of World Ocean Atlas 2009 climatology (monthly average) (WOA09) (black lines) and conductivity-temperature-depth (CTD) casts for the sediment trap during 2008 (blue lines), 2009 (red lines), 2010 (green lines), and 2011 (brown lines).

Sediment-trap samples were wet split into four aliquots using a precision rotary splitter at the University of South Carolina, stored in buffered deionized water, and then refrigerated. A quarter split was wet sieved over a 150- $\mu \mathrm{m}$ sieve and subsequently wet picked for all foraminifers. In samples with less than 300 foraminifers in the first quarter split, an additional one-quarter split was processed. The counts were then summed. All planktic foraminifers were identified to species. The species counts are reported as flux in tests per square meter per day. Flux was calculated by multiplying the individual species counts by number of splits, then dividing by sampling length, which was typically 7 or 14 days. Percent abundance (percent of each species within each cup) is reported weekly and for each season. 
Seasonal flux is the total flux for each individual species. Seasons are defined as winter (January, February, and March), spring (April, May, and June), summer (July, August, and September), and fall (October, November, and December). Relative seasonal abundances were calculated by dividing the individual species total seasonal flux by the total seasonal flux for all planktic foraminifers.

\section{Results from 2011}

From the sediment-trap material, 10 species, or varieties, of planktic foraminifers constituted 92 percent of the 2011 assemblage: Globigerinoides ruber (d'Orbigny) (pink and white varieties), Gs. sacculifer (Brady), Globigerina calida Parker, Globigerinella aequilateralis (Brady), Globorotalia menardii group [The Gt. menardii group includes Gt. menardii (Parker, Jones, and Brady), Gt. tumida (Brady), and Gt. ungulata Bermudez], Orbulina universa d'Orbigny, Gt. truncatulinoides (d'Orbigny), Pulleniatina spp., and Neogloboquadrina dutertrei (d'Orbigny) (table 1; figs. 4 and 5).

The mean daily flux of planktic foraminifers recovered from the trap in 2011 was 205 tests m$^{-2}$ day $^{-1}$ (table 1; fig. 4). The winter (January, February, and March) flux contributed about 36 percent of

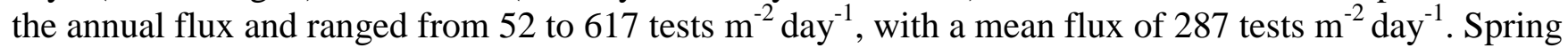
(April, May, and June) flux contributed about 17 percent of the annual flux and ranged from 48 to 174 tests $\mathrm{m}^{-2}$ day $^{-1}$, with a mean flux of 121 tests $\mathrm{m}^{-2}$ day $^{-1}$. Summer (July, August, and September) flux contributed about 16 percent of the annual flux and ranged from 72 to 672 tests $\mathrm{m}^{-2}$ day $^{-1}$, with a mean flux of 207 tests $\mathrm{m}^{-2} \mathrm{day}^{-1}$. The fall (October, November, and December) flux contributed about 32 percent of the annual flux and ranged from 62 to 565 tests $\mathrm{m}^{-2}$ day $^{-1}$, with a mean flux of 213 tests $\mathrm{m}^{-2}$ day $^{-1}$.

Globortotalia truncatulinoides was the most abundant species in the early winter season, comprising >62 percent of the assemblage during January and February (fig. 5). The spinose species Globigerinoides ruber (pink) (23 percent), Globigerinoides ruber (white) (17 percent), Globigerinoides sacculifer (9 percent), Globigerina calida (9 percent), Globigerinella aequilateralis (8 percent), the non-spinose Neogloboquadrina dutertrei (10 percent), and the Globorotalia menardii group ( 8 percent) accounted for the bulk of the spring percentage ( $~ 84$ percent). The summer season was dominated by the Globigerinoides genus (Gs. ruber (pink), Gs. ruber (white), and Gs. sacculifer), which made up 79 percent of the total assemblage. Gs. ruber (pink) (16 percent), Gs. ruber (white) (19 percent), Gs. sacculifer (15 percent), Gl. aequilateralis (9 percent), N. dutertrei (10 percent), Pulleniatina spp. (8 percent) and the Gt. menardii group (10 percent) accounted for $\sim 88$ percent of the fall assemblage.

Globigerinoides (Gs. ruber (pink), Gs. ruber (white), and Gs. sacculifer) flux (tests $\mathrm{m}^{-2} \mathrm{day}^{-1}$ ) increased by a factor of 2 from the average 2008-10 data to late September and early October 2011 data. The increase accounted for $\sim 89$ percent of the assemblage in late September and $\sim 87$ percent in early October. 


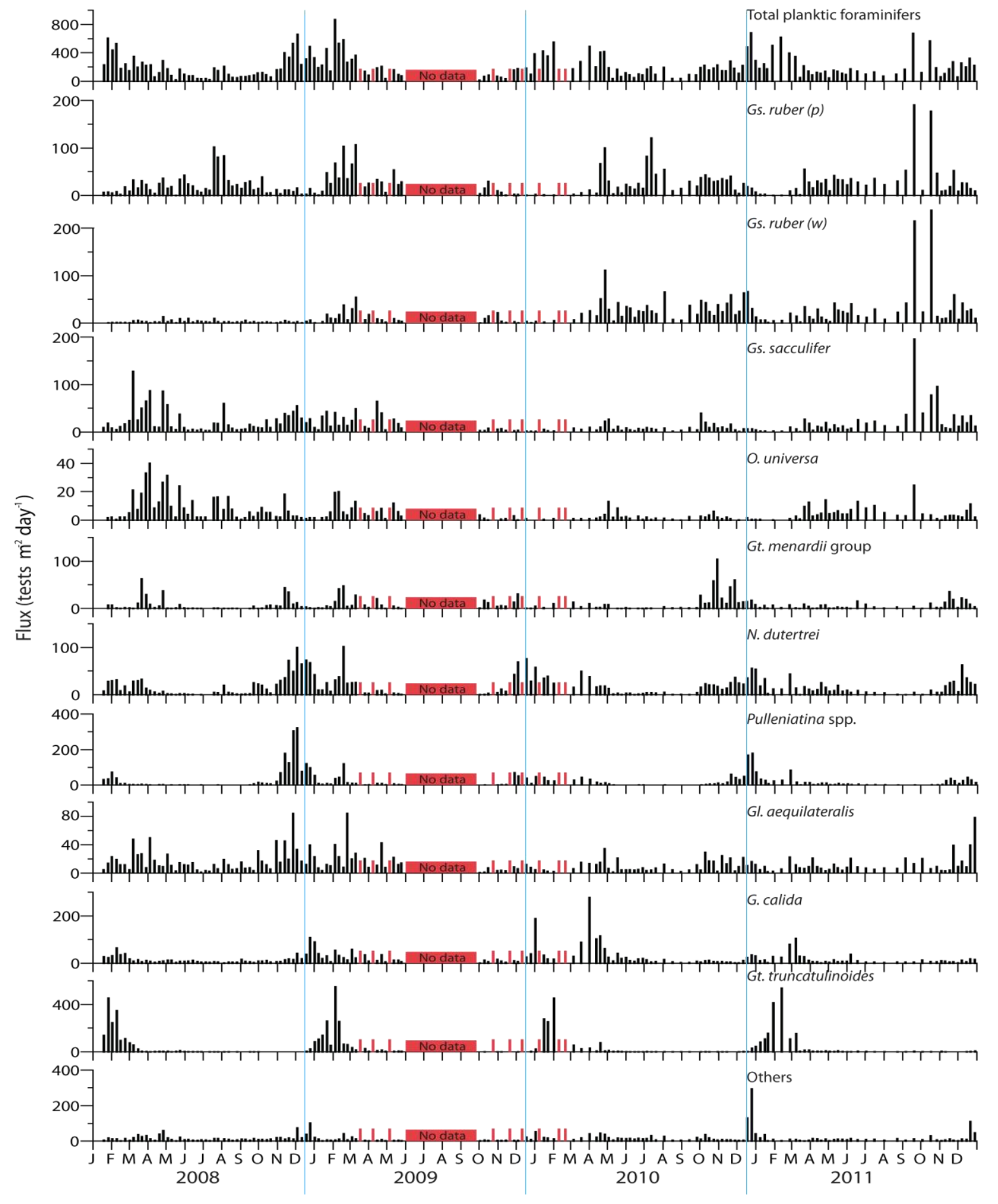

Figure 4. Average daily flux of 7- to 14-day-long sampling intervals of all planktic foraminifers and the 10 most abundant species/groups during 2008-11. Note the scale change in the $y$-axis. The tick marks on the $x$-axis denote the $1^{\text {st }}$ day of each month. There was a gap in sampling from late May to late September 2009 (red rectangles and designated as "No data") and loss of nine sample cups (red bars). $\mathrm{m}^{-2}$ day-1, square meter per day. 


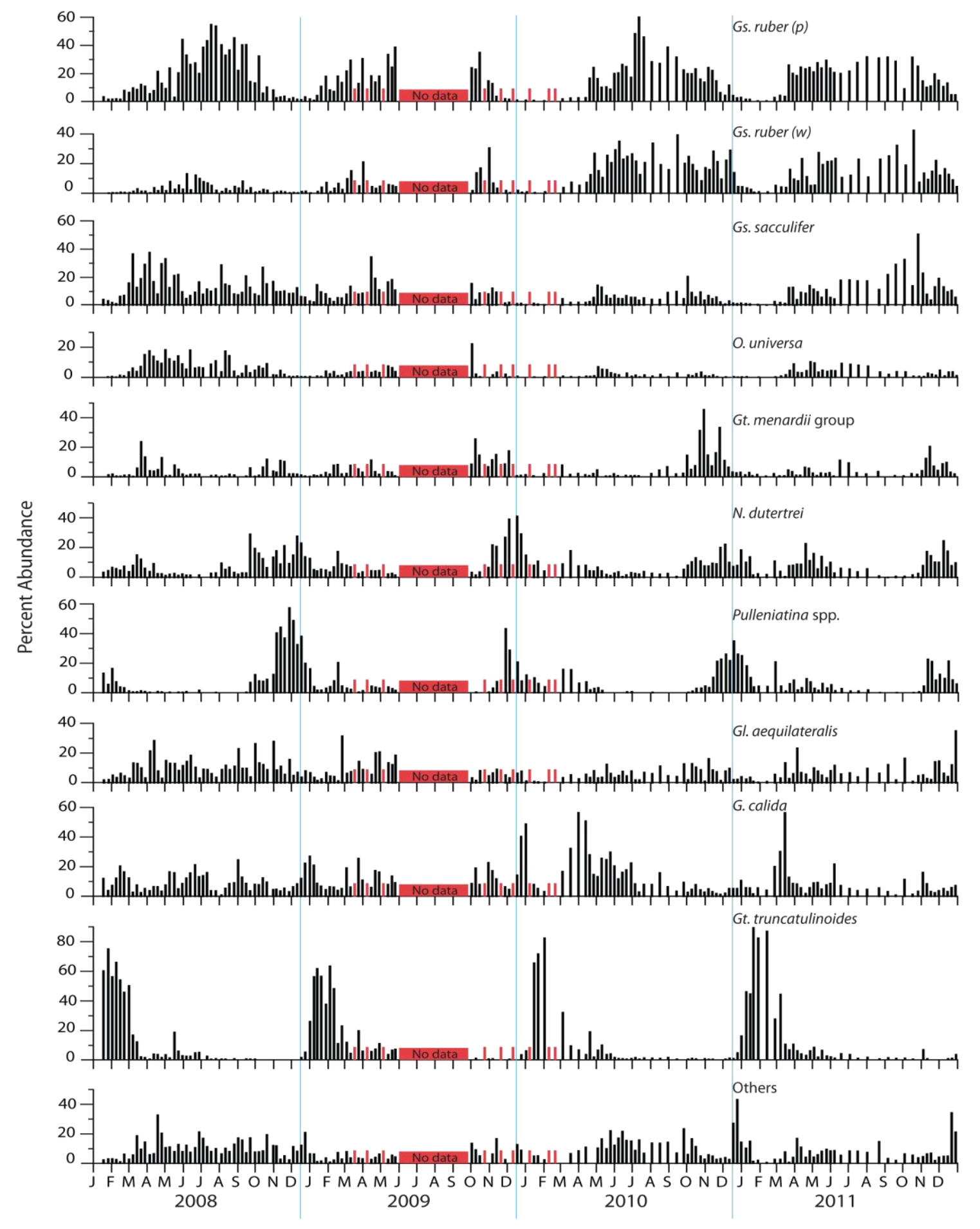

Figure 5. Weekly percent abundance of the 10 most common species/groups of planktic foraminifers during 2008-11. Note the scale change in the $y$-axis. The tick marks on the $x$-axis denote the 1 st day of each month. There was a gap in sampling from late May to late September 2009 (red rectangles and designated as "No data") and loss of nine sample cups (red bars). 


\section{Discussion}

The sediment record has shown that Gs. ruber (white) is a major component ( 20-30 percent) of the late Holocene planktic foraminifer assemblages (Kennett and others, 1985; LoDico and others, 2006; Poore and others, 2011). However, in this study, Gs. ruber (white) has been anomalously low (1.5 percent (2008), $\sim 5$ percent (2009), and $~ 14$ percent (2010 and 2011)). The addition of 2011 data to the 2008-10 flux and percentage data provide for a better understanding of the inter- and intra- annual variability. A longer time series is needed to distinguish the natural range of all foraminiferal species.

\section{Conclusions}

Sediment-trap data collected from January 2008 to December 2011 shows that 10 species/groups of planktic foraminifers comprised $\sim 92$ percent of the total flux. Globorotalia truncatulinoides flux data indicate that the species almost exclusively is present in January and February.

\section{Acknowledgments}

We thank Don Hickey, Eric Tappa, Katie Richwine, Joe Malbrough, and the crew of the R/V Pelican for assistance in deploying and redeploying the sediment trap mooring.

\section{References Cited}

Antonov, J.I., Seidov, D., Boyer, T.P., Locarnini, R.A., Mishonov, A.V., Garcia, H.E., Baranova, O.K., Zweng, M.M., and Johnson, D.R., 2010, World ocean atlas 2009, Volume 2: Salinity, in Levitus, S., ed., NOAA atlas NESDIS 69: Washington, D.C., U.S. Government Printing Office, 184 p.

Kennett, J.P., Elmstrom, K., and Penrose, N.L., 1985, The last deglaciation in Orca Basin, Gulf of Mexico: High-resolution planktonic foraminifera changes: Palaeogeography, Paleoclimatology, Paleoecology, v. 50, p. 189-216.

Locarnini, R.A., Mishonov, A.V., Antonov, J.I., Boyer, T.P., Garcia, H.E., Baranova, O.K., Zweng, M.M., and Johnson, D.R., 2010, World ocean atlas 2009, Volume 1: Temperature, in Levitus, S., ed., NOAA atlas NESDIS 68: Washington, D.C., U.S. Government Printing Office, 184 p.

LoDico, J.M., Flower, B.P., and Quinn, T.M., 2006, Subcentennial-scale climatic and hydrologic variability in the Gulf of Mexico during the early Holocene: Paleoceanography, v. 21, doi:10.1029/2005PA001243.

Poore, R.Z., Tedesco, K.A., and Spear, J.W., 2013, Seasonal flux and assemblage composition of planktic foraminifers from a sediment-trap study in the northern Gulf of Mexico, in Brock, J.C., Barras, J.A., and Williams, S.J., eds., Understanding and predicting change in the coastal ecosystems of the northern Gulf of Mexico: Journal of Coastal Research, Special Issue No. 63, p. 6-19.

Poore, R.Z., Verardo, S., Caplan, J., Pavich, K., and Quinn, T., 2011, Planktic foraminiferal relative abundance trends in the Gulf of Mexico Holocene sediments-Records of climate variability, in Holmes, C., ed., Gulf of Mexico-Its origins, waters, biota, and human impact: University Texas Press.

Tedesco, K.A., Spear, J.W., Tappa, Eric, and Poore, R.Z., 2009, Seasonal flux and assemblage composition of planktic foraminifera from the northern Gulf of Mexico: U.S. Geological Survey Open-File Report 2009-1293, 19 p. 
Wang, Chunzai, and Enfield, D.B., 2001, The tropical Western Hemisphere Warm Pool: Geophysical Research Letters, v. 28, p. 1635-1638.

Wang, Chunzai, Enfield, D.B., Lee, Sang-ki, and Landsea, C.W., 2006, Influences of the Atlantic Warm Pool on Western Hemisphere summer rainfall and Atlantic hurricanes: Journal of Climate, v. 19, p. 3011-3028.

Table 1. Planktic foraminiferal flux (tests per square meter per day, $\mathrm{m}^{-2} \mathrm{~d}^{-1}$ ) and percent contribution (in parentheses) to the total assemblage for the 14 most common species, northern Gulf of Mexico. Table is separated by season (winter, spring, summer, and fall) and year. The first 10 species listed comprised about 90 percent of the total flux. Particularly low fluxes $\left(<50\right.$ tests $\left.\mathrm{m}^{-2} \mathrm{~d}^{-1}\right)$ are denoted with an asterisk next to the mid-week collection date. 
Mid-week

\begin{tabular}{|c|c|c|c|c|c|c|c|c|c|c|}
\hline \multicolumn{2}{|c|}{ Sample } & \multirow[b]{2}{*}{ 18-Jan-08 } & \multicolumn{2}{|c|}{ Gs. ruber (pink) } & \multicolumn{2}{|c|}{ (white) } & \multicolumn{2}{|c|}{ Gs. sacculifer } & \multicolumn{2}{|c|}{ group } \\
\hline GMT-1 & 1 & & 6.86 & $(2.99)$ & 0.00 & $(0.00)$ & 9.14 & (3.99) & 0.00 & $(0.00)$ \\
\hline MT-1 & 2 & 25-Jan-08 & 7.43 & (1.23) & 0.57 & $(0.09)$ & 18.29 & (3.03) & 7.43 & (1.23) \\
\hline IT- -1 & 3 & eb-08 & 5.71 & $(1.31$ & 1.14 & $(0.26$ & 8.00 & $(1.8$ & 7.43 & $(1.7$ \\
\hline MT-1 & 4 & 8-Feb-08 & 8.00 & (1.52) & 1.14 & $(0.22)$ & 4.57 & (0.87) & 1.71 & $(0.33)$ \\
\hline GMT-1 & 5 & 15-Feb-08 & 2.29 & (1.29) & 1.14 & (0.65) & 10.86 & (6.13) & 0.57 & $(0.32)$ \\
\hline GMT-1 & 6 & 22-Feb-08 & 18.29 & (7. & 1.14 & $(0.47)$ & 16.57 & $(6.81)$ & 2.29 & $(0.94)$ \\
\hline GMT-1 & 7 & 29-Feb-08 & 9.14 & (6.15) & 0.57 & (0.38) & 23.43 & (15.77) & 1.71 & (1.15) \\
\hline MT-1 & 8 & aar-08 & 32.57 & (9.31) & 4.57 & (1.31) & 127.43 & (36.44) & 0.57 & $(0.16)$ \\
\hline GMT-1 & 9 & 14-Mar-08 & 16.00 & (8.16) & 5.71 & (2.92) & 24.57 & (12.54) & 11.43 & (5.83) \\
\hline GMT-1 & 10 & Mar-08 & 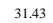 & 1.88) & 3.43 & 1.30) & 49.71 & (18.79) & 62.86 & (23.76) \\
\hline GMT-1 & 11 & 28-Mar-08 & 23.43 & 0.54) & 2.86 & 29) & 64.57 & (29.05) & 29.71 & (13.37) \\
\hline
\end{tabular}

\begin{tabular}{rrrrrrrrrrr}
\hline GMT-1 & 12 & 4-Apr-08 & 12.00 & $(5.22)$ & 0.57 & $(0.25)$ & 86.29 & $(37.56)$ & 9.14 & $(3.98$ \\
GMT-1 & 13 & 11-Apr-08 & 4.57 & $(7.41)$ & 2.29 & $(3.70)$ & 10.29 & $(16.67)$ & 2.29 & $(3.70)$
\end{tabular}

$\begin{array}{lllrlrlrrrr}\text { GMT-1 } & 13 & \text { 11-Apr-08 } & 4.57 & (7.41) & 2.29 & (3.70) & 10.29 & (16.67) & 2.29 & (3.70) \\ \text { GMT-1 } & 14 & \text { 18-Apr-08 } & 25.33 & (21.17) & 2.00 & (1.67) & 9.33 & (7.80) & 5.67 & (4.74)\end{array}$

$\begin{array}{rccccccrrrr}\text { GMT-1 } & 14 & 18 \text {-Apr-08 } & 25.33 & (21.17) & 2.00 & (1.67) & 9.33 & (7.80) & 5.67 & (4.74) \\ \text { GMT-2 } & 1 & \text { 25-Apr-08 } & 36.57 & (12.61) & 13.43 & (4.63) & 85.71 & (29.56) & 37.43 & (12.91)\end{array}$

$\begin{array}{lllllllllllll}\text { GMT-2 } & 2 & 2-M a y-08 & 15.43 & (8.91) & 2.86 & (1.65) & 57.14 & (33.00) & 1.14 & (0.66) & 0\end{array}$

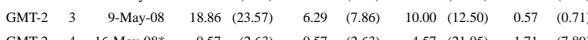

$\begin{array}{lllllllllll}\text { GMT-2 } & 4 & 16-M a y-08^{*} & 0.57 & (2.63) & 0.57 & (2.63) & 4.57 & (21.05) & 1.71 & (7.89) \\ \end{array}$

$\begin{array}{lllllllllll}\text { GMT-2 } & 5 & 23-M a y-08 & 34.29 & (20.13) & 9.14 & (5.37) & 37.14 & (21.81) & 8.57 & (5.03)\end{array}$

$\begin{array}{lllllllllll}\text { GMT-2 } & 7 & \text { 6-Jun-08 } & 24.57 & (32.82) & 9.71 & (12.98) & 3.43 & (4.58) & 0.57 & (0.76\end{array}$

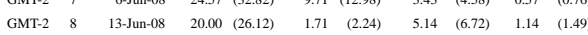

$\begin{array}{lcccccccccccccccccccc}\text { GMT-2 } & 8 & 13-J u n-08 & 20.00 & (26.12) & 1.71 & (2.24) & 5.14 & (6.72) & 1.14 & (1.49) & 0.00 & (0.00) & 1.14 & (1.49) & 14.29 & (18.66) & 12.00 & (15.67) & 13.71 & (17.91 \\ \text { GMT-2 } & 9 & \text { 20-Jun-08* } & 10.29 & (27.27) & 4.57 & (12.12) & 3.43 & (9.09) & 0.57 & (1.52) & 0.00 & (0.00) & 0.00 & (0.00) & 4.00 & (10.61) & 8.00 & (21.21) & 2.29 & (6.06)\end{array}$

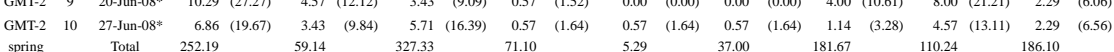

\begin{tabular}{|c|c|c|c|c|c|c|c|c|c|c|c|c|c|c|c|c|c|c|c|c|}
\hline & & \multirow{2}{*}{ (\%) } & \multirow[b]{2}{*}{ 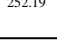 } & \multirow[b]{2}{*}{ (17.18) } & \multirow{2}{*}{9.14} & & & & & & \multirow[b]{2}{*}{$(0.36)$} & & & & \\
\hline & & & & & & $(4.03)$ & & (22.30) & & & & & & (2.52) & & (12.38) & & .51) & & (12.68) \\
\hline $\mathrm{AT}-2$ & 11 & 4-Jul-08 & 14.29 & $(38.46)$ & 2.86 & $(7.69)$ & 2.86 & $(7.69)$ & 0.00 & 00) & 0.00 & $(0.00)$ & 0.00 & $(0.00)$ & 3.43 & 23) & 4 & 85) & 29 & \\
\hline T-2 & 12 & 11-Jul-08 & 10.86 & (43.18) & 1.71 & .82) & 2.86 & (11.36) & 0.00 & $(0.00)$ & 0.00 & $(0.00)$ & 0.0 & $(0.00)$ & 2.29 & (9.09) & 0 & (15.91) & 1.00 & $(0.00$ \\
\hline $\mathrm{T}-2$ & 13 & & 2.00 & & 9.7 & & 29 & $(9.82$ & 3 & & 00 & $(0.00)$ & 14 & 76) & 11.71 & (6.29) & 86 & 68) & .00 & 8.55 \\
\hline $4 \mathrm{~T}-2$ & 14 & Jul-08 & 7 & ( & 2.86 & (1.90) & 43 & (11.60 & 1.43 & $(0.95)$ & 0.29 & (0.19) & 4.57 & 3.044 & $3 . / 1$ & $(3.80)$ & 49 & 1.61) & 16.29 & 10.84 \\
\hline & 1 & $-t$ & & & & & & (2) & 7 & & & 0) & 20.00 & 64) & 18.86 & $(9.09)$ & 71 & 83) & 7.43 & $(3.5$ \\
\hline IT-3 & 2 & & & & & (2.98) & 14.29 & (14.8 & 29 & $(0.30)$ & 0.00 & $(0.00)$ & 5.14 & 36) & 11.43 & (11.90) & 14 & .36) & 16.57 & (17.26) \\
\hline $17-3$ & 3 & $\lg -0$ & 71 & s & 0.86 & (1.59) & .43 & (13.7 & 0.86 & (1.59) & 0.00 & $(0.00)$ & 3.71 & (6.88) & 4.86 & $(8.9$ & 4.57 & 47) & 71 & (14.29 \\
\hline & 4 & & 86 & & & & 0 & & 7 & & v & $(0.00)$ & 11 & 9) & 5.71 & (11.30) & 37 & $9.047)$ & 2.00 & 5.9 \\
\hline & 5 & & & & & & & & 0 & & & & & & 15.43 & (23.18) & 29 & (24.46) & 0.57 & 0. \\
\hline IT -3 & 6 & p-08 & 86 & ( & 5.43 & (8.12) & 6.00 & 8.97) & 00 & $(0.00)$ & 00 & 10) & 2.00 & 99) & 6.57 & (9.83) & 8.57 & (12.82) & 1.71 & $(2.56$ \\
\hline GMT-3 & 7 & sер-бол & 30.57 & (40.38 & 1.4 & (1.89) & 15.71 & (20.7 & 9 & $(0.38)$ & 9 & $(0.38)$ & 2.29 & 12) & 7.43 & (9.81) & 6.57 & .68) & 5.71 & 7.5 \\
\hline & 8 & & 12.00 & & 3. & & 10.86 & & & & 0.00 & (6.99) & 5.14 & (4) & 3.43 & (5.97) & 14 & (4) & 2.57 & .50 \\
\hline T-3 & 9 & 29 - & & 2.96) & 0.86 & 173 & 9.14 & (7.82) & 0.29 & .24) & & 2.22) & 86 & .56) & 31. & (26.65) & & $(8.07)$ & & \\
\hline mmer & & & 4.29 & & 1.57 & & 3.14 & & 86 & & 186 & & 4.00 & & 8.00 & & 1.43 & & 4.29 & \\
\hline
\end{tabular}

$\begin{array}{rrrrrrrrrrr}(3.16) & (14.20) & (0.89) & (1.71) & (7.71) & (10.50) & (6.68) & (6.91)\end{array}$

$\begin{array}{lllllllllllllllllllll}\text { GMT-3 } & 11 & 13-\text { Oct-08 } & 5.14 & (5.57) & 2.29 & (2.48) & 24.86 & (26.93) & 6.00 & (6.50) & 7.14 & (7.74) & 11.71 & (12.69) & 11.43 & (12.38) & 11.43 & (12.38) & 5.43 & (5.88\end{array}$

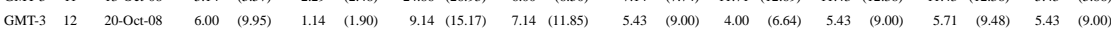

$\begin{array}{rccccccccccrrrrrrrrrr}\text { GMT-3 } & 12 & 20-\text { Oct-08 } & 6.00 & (9.95) & 1.14 & (1.90) & 9.14 & (15.17) & 7.14 & (11.85) & 5.43 & (9.00) & 4.00 & (6.64) & 5.43 & (9.00) & 5.71 & (9.48) & 5.43 & (9.00) \\ \text { GMT-4 } & 1 & 30-\text { Oct-08 } & 12.57 & (7.80) & 0.57 & (0.35) & 27.14 & (16.84) & 6.00 & (3.72) & 20.00 & (12.41) & 22.00 & (13.65) & 45.43 & (28.19) & 7.14 & (4.43) & 2.57 & (1.60)\end{array}$

$\begin{array}{llrrlllllllllllllllll}\text { GMT-4 } & 1 & 30-\text {-Oct-08 } & 12.57 & (7.80) & 0.57 & (0.35) & 27.14 & (16.84) & 6.00 & (3.72) & 20.00 & (12.41) & 22.00 & (13.65) & 45.43 & (28.19) & 7.14 & (4.43) & 2.57 & (1.60) \\ \text { GMT-4 } & 2 & 5-\text { Nov-08 } & 4.00 & (2.36) & 1.71 & (1.01) & 16.00 & (9.46) & 5.14 & (3.04) & 68.57 & (40.54) & 30.29 & (17.91) & 14.86 & (8.78) & 8.00 & (4.73) & 2.29 & (1.35)\end{array}$

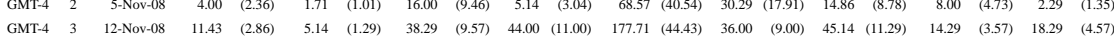

$\begin{array}{lllllllllllllllllllll}\text { GMT-4 } & 4 & 19-\mathrm{Nov}-08 & 11.43 & (3.39) & 2.86 & (0.85) & 33.71 & (10.00) & 34.86 & (10.34) & 125.14 & (37.12) & 72.00 & (21.36) & 19.43 & (5.76) & 18.29 & (5.42) & 6.29 & (1.86\end{array}$

$\begin{array}{lrrrrrrrrrrrrrrrrrrrr}\text { GMT-4 } & 5 & 26-N o v-08 & 8.00 & (1.51) & 1.14 & (0.22) & 42.86 & (8.10) & 9.71 & (1.84) & 305.14 & (57.67) & 49.14 & (9.29) & 84.00 & (15.87) & 15.43 & (2.92) & 2.86 & (0.54) \\ \text { GMT-4 } & 6 & \text { 3-Dec-08 } & 16.00 & (2.43) & 2.86 & (0.43) & 54.86 & (8.32) & 12.57 & (1.91) & 322.86 & (48.96) & 99.43 & (15.08) & 33.14 & (5.03) & 41.71 & (6.33) & 2.86 & (0.43)\end{array}$

$\begin{array}{lllllllllllllllllllll}\text { GMT-4 } & 6 & 3 \text {-Dec-08 } & 16.00 & (2.43) & 2.86 & (0.43) & 54.86 & (8.32) & 12.57 & (1.91) & 322.86 & (48.96) & 99.43 & (15.08) & 33.14 & (5.03) & 41.71 & (6.33) & 2.86 & (0.43)\end{array}$

$\begin{array}{lllllllllllllllllllll}\text { GMT-4 } & 7 & 10-\text { Dec-08 } & 2.86 & (1.23) & 0.57 & (0.25) & 28.57 & (12.29) & 4.00 & (1.72) & 76.00 & (32.68) & 64.57 & (27.76) & 16.57 & (7.13) & 19.43 & (8.35) & 1.71 & (0.74) \\ \text { GMT-4 } & 8 & 17-\text { Dec-08 } & 286 & (0.91) & 3.43 & (1.09) & 18.86 & (6.01 & 3.43 & (1.09) & 120.00 & (38.25 & 72.57 & (23.13) & 12.00 & (3.83) & 37.71 & (1202) & 1.14 & (0.36)\end{array}$

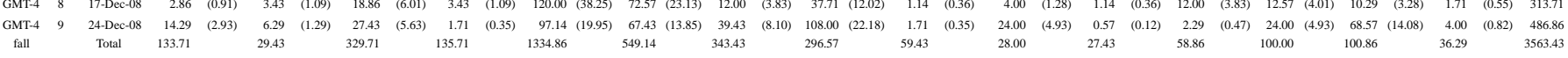

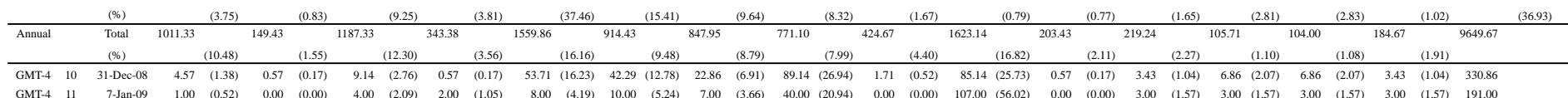

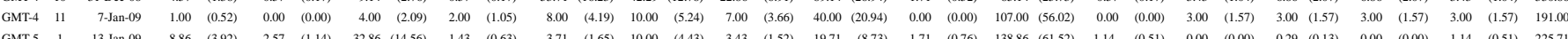
$\begin{array}{llllllllllllllllllllllllllllllllll}\text { GMT-5 } & 1 & 13-J a n-09 & 8.86 & (3.92) & 2.57 & (1.14) & 32.86 & (14.56) & 1.43 & (0.63) & 3.71 & (1.65) & 10.00 & (4.43) & 3.43 & (1.52) & 19.71 & (8.73) & 1.71 & (0.76) & 138.86 & (61.52) & 1.14 & (0.51) & 0.00 & (0.00) & 0.29 & (0.13) & 0.00 & (0.00) & 1.14 & (0.51) & 225.71 \\ \text { GMT-5 } & 2 & \text { 20-Jan-09 } & 48.00 & (10.49) & 18.29 & (4.00) & 42.86 & (9.36) & 5.71 & (1.25) & 8.00 & (1.75) & 25.14 & (5.49) & 12.00 & (2.62) & 30.86 & (6.74) & 2.29 & (0.50) & 257.71 & (56.30) & 0.00 & (0.00) & 4.00 & (0.87) & 0.00 & (0.00) & 0.00 & (0.00) & 2.86 & (0.62) & 457.71\end{array}$

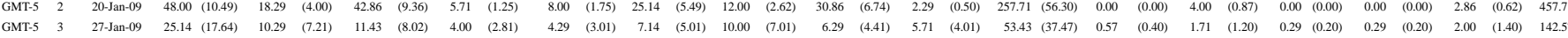
$\begin{array}{llllllllllllllllllllllllllllllllll}\text { GMT-5 } & 4 & 3-F e b-09 & 68.00 & (7.86) & 8.57 & (0.99) & 40.57 & (4.69) & 14.86 & (1.72) & 35.43 & (4.10) & 30.86 & (3.57) & 40.00 & (4.62) & 54.29 & (6.27) & 19.43 & (2.25) & 546.86 & (63.21) & 1.71 & (0.20) & 2.86 & (0.33) & 0.00 & (0.00) & 0.00 & (0.00) & 1.71 & (0.20) & 865.14\end{array}$ $\begin{array}{llllllllllllllllllllllllllllllllll}\text { GMT-5 } & 5 & 10-\text { Feb-09 } & 36.57 & (6.89) & 17.71 & (3.34) & 13.14 & (2.48) & 41.71 & (7.86) & 42.86 & (8.07) & 37.71 & (7.10) & 22.86 & (4.31) & 32.57 & (6.14) & 20.00 & (3.77) & 254.29 & (47.90) & 4.00 & (0.75) & 4.57 & (0.86) & 0.00 & (0.00) & 0.57 & (0.11) & 2.29 & (0.43) & 530.86\end{array}$ $\begin{array}{llllllllllllllllllllllllllllllllll}\text { GMT-5 } & 6 & 17-F e b-09 & 103.43 & (17.80) & 37.71 & (6.49) & 30.29 & (5.21) & 48.00 & (8.26) & 118.86 & (20.45) & 101.14 & (17.40) & 8.00 & (1.38) & 22.86 & (3.93) & 5.71 & (0.98) & 62.86 & (10.82) & 0.57 & (0.10) & 32.57 & (5.60) & 7.43 & (1.28) & 0.00 & (0.00) & 1.71 & (0.29) & 581.14\end{array}$

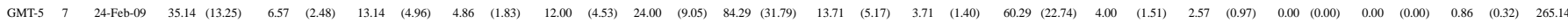

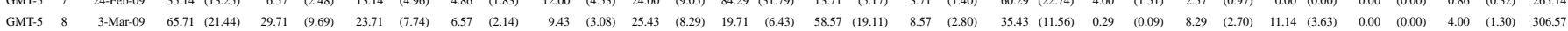

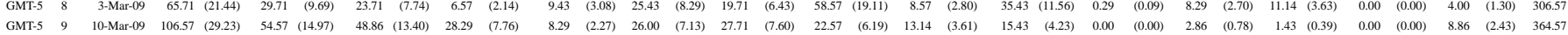

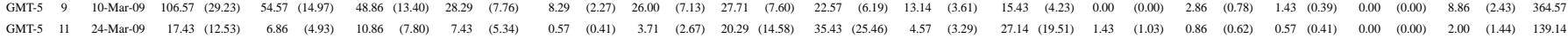

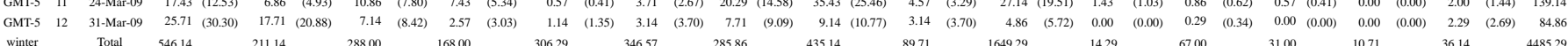

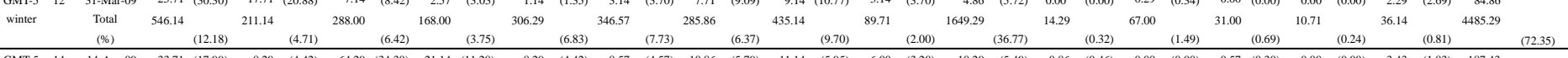

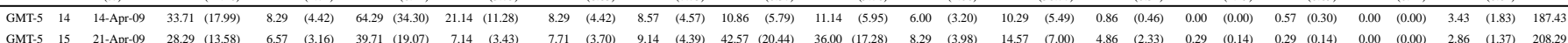
$\begin{array}{rrrrrrrrrrrrrrrrrrrrrrrrrrrrrrrrrr}\text { GMT-5 } & 15 & 21 \text {-Apr-09 } & 28.29 & (13.58) & 6.57 & (3.16) & 39.71 & (19.07) & 7.14 & (3.43) & 7.71 & (3.70) & 9.14 & (4.39) & 42.57 & (20.44) & 36.00 & (17.28) & 8.29 & (3.98) & 14.57 & (7.00) & 4.86 & (2.33) & 0.29 & (0.14) & 0.29 & (0.14) & 0.00 & (0.00) & 2.86 & (1.37) & 208.29 \\ \text { GMT-5 } & 16 & 28 \text {-Apr-09* } & 6.57 & (17.83) & 1.71 & (4.65) & 4.00 & (10.85) & 0.57 & (1.55) & 1.14 & (3.10) & 1.71 & (4.65) & 7.71 & (20.93) & 6.00 & (16.28) & 1.14 & (3.10) & 4.00 & (10.85) & 0.86 & (2.33) & 0.00 & (0.00) & 0.00 & (0.00) & 0.00 & (0.00) & 1.43 & (3.88) & 36.86\end{array}$

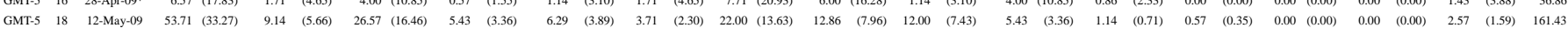

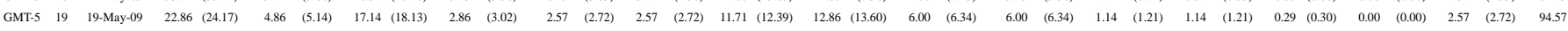

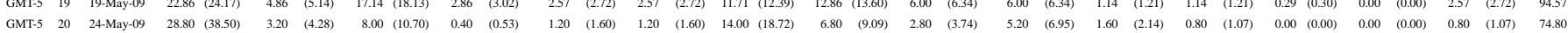

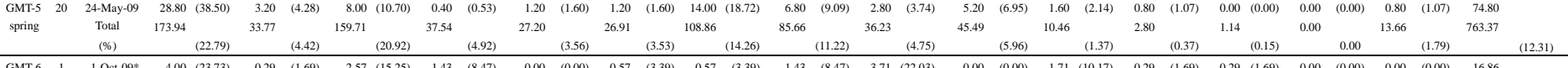

\begin{tabular}{|c|c|c|c|c|c|c|c|c|c|c|c|c|c|c|c|c|c|c|c|c|c|c|c|c|c|c|c|c|c|c|c|c|c|c|}
\hline & & (2) & & (22.79) & & (4.42) & & $0.92)$ & & (4.92) & & (3.56) & & (3.53) & & (14.26) & & (11.22) & & (4.75) & & (5.96) & & (1.37) & & $(0.37)$ & & $(0.15)$ & & .00 & & 1.79] & & (12.31) \\
\hline GMT-6 & 1 & 1-Oct-09* & 4.00 & (23.73) & 0.29 & (1.69) & 2.57 & (15.25) & 1.43 & $(8.47)$ & 0.00 & $(0.00)$ & 0.57 & (3.39) & 0.57 & (3.39) & 1.43 & $(8.47)$ & 3.71 & (22.03) & 0.00 & $(0.00)$ & 1.71 & (10.17) & 0.29 & (1.69) & 0.29 & (1.69) & 0.00 & $(0.00)$ & 0.00 & $(0.00)$ & 16.86 & \\
\hline GMT-6 & 2 & Oct-0s & 71 & 73) & 9.43 & (13.64) & 2.57 & (3.72) & 1.71 & (25.62) & 0.29 & $(0.41$ & 1.14 & 65) & 14 & .65) & 13.14 & (19.01) & 1.43 & (2.07) & 0.57 & (0.83) & 5.43 & (7.85) & 0.57 & (0.83) & 00 & $(0.6$ & 0 & .00) & 00 & $0.00)$ & 69.14 & \\
\hline MT-6 & 3 & 15-Oct- 09 & 30.00 & (34.77) & 14.57 & (16.89) & 7.43 & $(8.61)$ & 12.57 & (14.57) & 0.00 & $(0.00)$ & 3.14 & (3.64) & 7.14 & (8.28) & 6.86 & (7.95) & 0.29 & $(0.33)$ & 0.00 & $(0.00)$ & 2.00 & (2.32) & 0.57 & (0.66) & 0.00 & $(0.00)$ & 0.86 & $0.99)$ & 0.86 & (0.99) & 86.29 & \\
\hline IT-6 & 5 & 29-Oct-09 & 10.29 & (14.34) & 21.71 & (30.28) & 5.71 & $(7.97)$ & 4.86 & (6.77) & 0.29 & $(0.40)$ & 4.86 & $(6.77)$ & 3.43 & (4.78) & 16.29 & (22.71) & 0.00 & $(0.00)$ & 29 & $(0.40)$ & 0.57 & $(0.80)$ & 2.29 & (3.19) & 0.00 & $(0.00)$ & .00 & 0) & 1.14 & (1.59) & 71.71 & \\
\hline MT-6 & 6 & 5-Nov-09 & 6.86 & (12.50) & 3.71 & $(6.7$ & 6.57 & (11.98) & 6.29 & (11.46) & 1.71 & (3.13) & 12.00 & (21.88) & 3.71 & $(6.77)$ & 9.43 & (17.19 & 0.86 & (1.56) & 29 & $(0.52)$ & 0.57 & (1.04) & 2.29 & $(4.17)$ & 0.00 & $(0.00)$ & 00 & .00) & 0.57 & 1.04) & 54.86 & \\
\hline IT-6 & 7 & 12-Nov-09* & 1.14 & (3.33) & 1.14 & $(3.33$ & 3.14 & $(9.17)$ & 5.14 & (15.00) & 2.57 & (7.50) & 7.14 & (20.83) & 3.14 & $(9.17)$ & 4.00 & (11.67 & 1.14 & (3.33) & 0.00 & $(0.00)$ & 0.86 & (2.50) & 3.43 & (10.00) & 00 & ) & 0.00 & 00) & 1.43 & 4.17) & 34.2 & \\
\hline GMT-6 & 9 & Iov-09 & 2.57 & (1.62) & 2.86 & (1.80) & 2.29 & 4) & 71 & (8.62) & 69.14 & 43.45) & 42.86 & (26.93) & 8.57 & (5.39) & 10.00 & (6.28) & 3.14 & (1.5) & 0.00 & $(0.00)$ & 1.43 & (0.90) & 0.29 & $(0.18)$ & 6 & (0) & 0.29 & (0.18) & 1.14 & 0.72) & 159.14 & \\
\hline $\mathrm{T}-6$ & 10 & ec-09 & 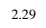 & (1.30) & 0. & & & (1.94) & .86 & (17.50) & 50.86 & (28.85) & 69.14 & (39 & 0 & (3. & 8.00 & (4.54) & 29 & $(0$. & & 16) & 2. & (1.30) & 00 & & 9 & 6) & 57 & & 43 & .81) & 176.29 & \\
\hline GMT-6 & 12 & 17-Dec-09 & 1.14 & $(0.62)$ & 3.43 & (1.86) & 1.71 & $(0.93)$ & 1.14 & $(0.62)$ & 38.29 & (20.74) & 76.00 & (41.18) & 12.00 & (6.50) & 26.29 & (14.24) & 1.14 & $(0.62)$ & 0.00 & $(0.00)$ & 0.57 & $(0.31)$ & 1.14 & $(0.62)$ & 3.43 & (1.86) & 17.71 & $(9.60)$ & 0.57 & $(0.31)$ & 184.57 & \\
\hline GMT-6 & 13 & 24-Dec-09 & 0.00 & $(0.00)$ & 0.57 & $(0.58)$ & 1.14 & (1.17) & 0.86 & $(0.88)$ & 7.71 & (7.89) & 8.57 & (29.24) & 7.71 & $(7.89)$ & 39.43 & (40.35) & .00 & $(0.00)$ & 3.14 & $(3.22)$ & .43 & (1.46) & .00 & $(0.00)$ & 86 & $(2.92)$ & 3.71 & (3.80) & 57 & $(0.58)$ & 1.71 & \\
\hline \multirow{2}{*}{ fall } & & Total & 74.00 & & 58.29 & & 36.57 & & 94.57 & & 170.86 & & 245.43 & & 53.43 & & 134.86 & & 2.00 & & 4.57 & & 16.86 & & 10.86 & & 7.71 & & 23.14 & & 7.71 & & 950. & \\
\hline & & $(\%)$ & & $(7.78)$ & & $(6.13)$ & & (3.85) & & $(9.95)$ & & (17.97) & & $(25.81)$ & & (5.62) & & (14.18) & & $(1.26)$ & & $(0.48)$ & & (1.77) & & (1.14) & & $(0.81)$ & & (2.43) & & $(0.81)$ & & (15.34) \\
\hline
\end{tabular}

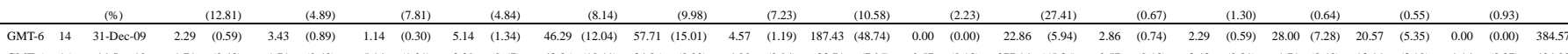
\begin{tabular}{lllllllllllllllllllllllllllllllllllll} 
GMT-6 & 16 & 14-Jan-10 & 1.71 & $(0.40)$ & 1.71 & $(0.40)$ & 5.14 & $(1.21)$ & 2.29 & $(0.67)$ & 42.86 & $(10.11)$ & 34.86 & $(8.22)$ & 4.00 & $(0.94)$ & 33.71 & $(7.95)$ & 0.57 & $(0.13)$ & 277.14 & $(65.36)$ & 0.57 & $(0.13)$ & 3.43 & $(0.81)$ & 1.71 & $(0.40)$ & 13.14 & $(3.10)$ & 1.14 & $(0.27)$ & 424.00 \\
\hline
\end{tabular} $\begin{array}{lllllllllllllllllllllllllllllllllll}\text { GMT-6 } & 17 & 21-\mathrm{Jan}-10 & 0.00 & 0.00 & 0.00 & (0.00) & 2.29 & (0.64) & 0.00 & (0.00) & 22.29 & (6.28) & 38.86 & (10.95) & 2.29 & (0.64) & 18.29 & (5.15) & 0.00 & (0.00) & 253.71 & (71.50) & 0.00 & (0.00) & 9.14 & (2.58) & 0.57 & (0.16) & 6.29 & (1.77) & 1.14 & (0.32) & 354.86 & \end{array}$ $\begin{array}{lllllllllllllllllllllllllllllllllllll}\text { GMT-6 } & 18 & 31-\text { Jan-10 } & 1.14 & (0.21) & 4.86 & (0.88) & 1.71 & (0.31) & 10.57 & (2.13) & 22.00 & (4.01) & 24.00 & (4.37) & 2.00 & (0.36) & 17.43 & (3.18) & 1.14 & (0.21) & 451.14 & (82.20) & 0.00 & (0.00) & 7.71 & (1.41) & 1.71 & (0.31) & 1.43 & (0.26) & 2.00 & (0.36) & 548.86\end{array}$ 


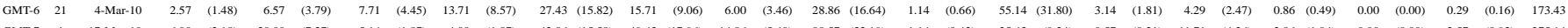
$\begin{array}{lllllllllllllllllllllllllllllllllll}\text { GMT-7 } & 2 & \text { 31-Mar-10 } & 6.00 & 11.43 & (2.34) & 25.71 & (5.25) & 9.43 & (1.93) & 10.57 & (2.39) & 31.43 & (6.42) & 38.00 & (7.76) & 13.43 & (2.74) & 275.71 & (56.33) & 1.14 & (0.23) & 31.43 & (6.42) & 3.14 & (0.64) & 21.71 & (4.44) & 13.43 & (2.74) & 0.00 & (0.00) & 2.86 & (0.58) & 489.43\end{array}$

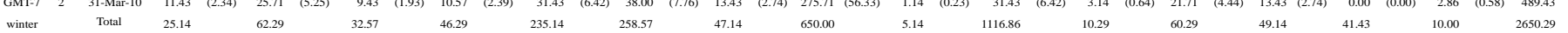

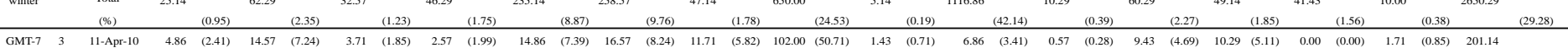

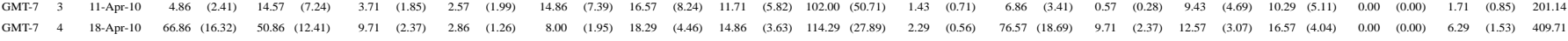

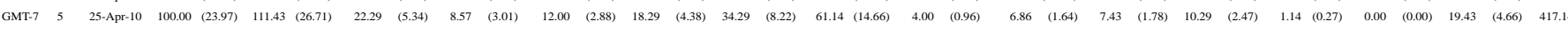

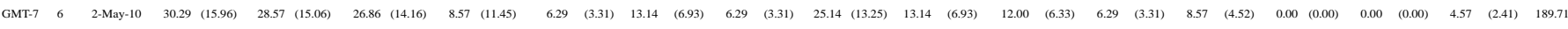
$\begin{array}{llllllllllllllllllllllllllllllllll}\text { GMT-7 } & 7 & 9-M a y-10^{*} & 3.71 & (9.77) & 4.00 & (10.53) & 4.86 & (12.78) & 0.00 & (5.26) & 0.57 & (1.50) & 1.71 & (4.51) & 1.43 & (3.76) & 9.71 & (25.56) & 2.00 & (5.26) & 3.71 & (9.77) & 5.71 & (15.04) & 0.29 & (0.75) & 0.00 & (0.00) & 0.00 & (0.00) & 0.29 & (0.75) & 38.00\end{array}$

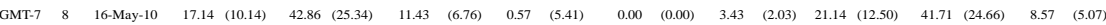

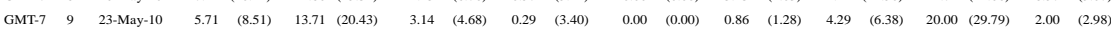
$\begin{array}{lllllllllllllllllllll}\text { GMT-7 } & 10 & 30-\text { May-10 } & 23.43 & (19.62) & 34.57 & (28.95) & 8.29 & (6.94) & 2.29 & (4.07) & 0.00 & (0.00) & 3.43 & (2.87) & 4.29 & (3.59) & 24.29 & (20.33) & 2.57 & (2.15)\end{array}$ $\begin{array}{lllllllllllllllllllll}\text { GMT-7 } & 11 & \text { 6-Jun-10 } & 18.29 & (20.19) & 31.43 & (34.70) & 4.29 & (4.73) & 0.57 & (2.21) & 0.00 & (0.00) & 3.43 & (3.79) & 4.57 & (5.05) & 10.57 & (11.67) & 1.43 & (1.58)\end{array}$ $\begin{array}{llllllllllllllllllllll}\text { GMT-7 } & 12 & 13-J u n-10 & 13.43 & (26.11) & 11.71 & (22.78) & 2.29 & (4.44) & 0.00 & (0.00) & 0.00 & (0.00) & 0.29 & (0.56) & 4.00 & (7.78) & 8.29 & (16.11) & 0.00 & (0.00\end{array}$

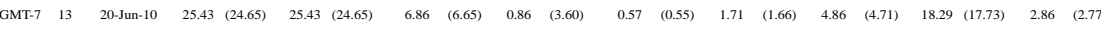
$\begin{array}{llllllllllllllllllllll}\text { GMT-7 } & 14 & 27-J u n-10 & 15.14 & (16.99) & 23.43 & (26.28) & 4.86 & (5.45) & 0.57 & (1.28) & 0.57 & (0.64) & 2.86 & (3.21) & 7.14 & (8.01) & 20.00 & (22.44) & 0.57 & (0.64)\end{array}$ $\begin{array}{lllllllllllll}5.71 & (3.38) & 4.57 & (2.70) & 6.86 & (4.05) & 0.00 & (0.00) & 0.00 & (0.00) & 5.14 & (3.04) & 169.14\end{array}$ $\begin{array}{lllllllllllll}2.57 & (3.83) & 7.71 & (11.49) & 3.43 & (5.11) & 0.00 & (0.00) & 0.00 & (0.00) & 3.43 & (5.11) & 67.14\end{array}$

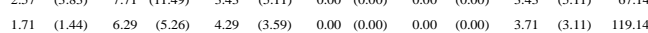
$\begin{array}{lllllllllllll}0.57 & (0.63) & 4.57 & (5.05) & 6.86 & (7.57) & 0.00 & (0.00) & 0.00 & (0.00) & 4.00 & (4.42) & 90.57\end{array}$ $\begin{array}{lllllllllllll}0.29 & (0.56) & 2.86 & (5.56) & 3.14 & (6.11) & 0.29 & (0.56) & 0.00 & (0.00) & 4.86 & (9.44) & 51.43\end{array}$ $\begin{array}{lllllllllllll}0.29 & (0.28) & 2.86 & (2.77) & 2.57 & (2.49) & 0.00 & (0.00) & 0.00 & (0.00) & 10.57 & (10.25) & 103.1\end{array}$ $\begin{array}{llllllllllllll}0.57 & (0.64) & 5.71 & (6.41) & 2.00 & (2.24) & 0.57 & (0.64) & 0.00 & (0.00) & 5.14 & (5.7) & 89.14\end{array}$

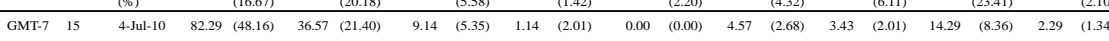

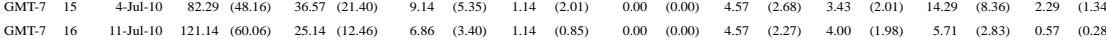

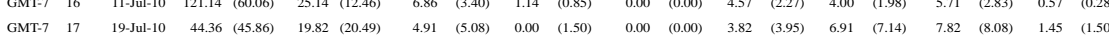

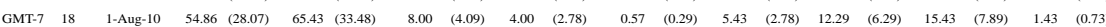
$\begin{array}{llllllllllllllllllllll}\text { GMT-7 } & 19 & 15 \text {-Aug-10* } & 10.43 & (26.84) & 7.43 & (19.12) & 1.57 & (4.04) & 1.71 & (6.25) & 0.00 & (0.00) & 0.86 & (2.21) & 4.43 & (11.40) & 6.14 & (15.81) & 0.71 & (1.84)\end{array}$

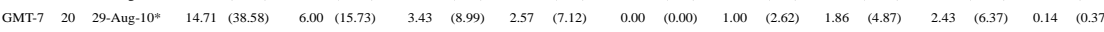

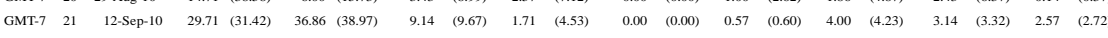

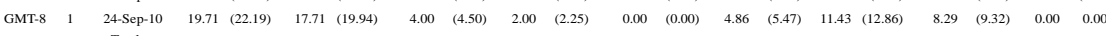
\begin{tabular}{llllllllll}
$(6.05)$ & $(3.30)$ & $(3.61)$ & $(1.48)$ & & 0.00 & & $(3.55)$ & $(21.49)$ \\
\hline
\end{tabular} $\begin{array}{lllllllllllll}.29 & (1.34) & 8.57 & (5.02) & 1.71 & (1.00) & 0.00 & (0.00) & 0.00 & (0.00) & 4.57 & (2.68) & 170.86\end{array}$ $\begin{array}{lllllllllllll}0.57 & (0.28) & 28.00 & (13.88) & 1.14 & (0.57) & 0.00 & (0.00) & 0.00 & (0.00) & 2.86 & (1.42) & 201.7 \\ 1.09 & (1.13) & 3.27 & (3.38) & 0.91 & (0.94) & 0.00 & (0.00) & 0.00 & (0.00) & 2.36 & (2.44) & 96.73\end{array}$ $\begin{array}{lllllllllllll}2.00 & (1.02) & 9.43 & (4.82) & 7.14 & (3.65) & 0.29 & (0.15) & 0.00 & (0.00) & 9.14 & (4.68) & 195.43\end{array}$ $\begin{array}{lllllllllllll}0.29 & (0.74) & 3.14 & (8.09) & 0.71 & (1.84) & 0.00 & (0.00) & 0.00 & (0.00) & 1.43 & (3.68) & 38.86\end{array}$

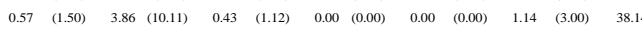

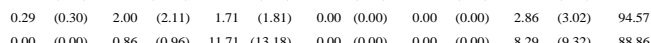

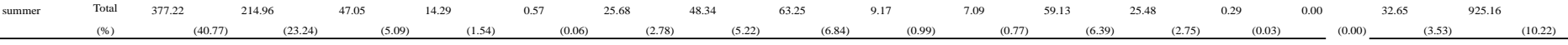

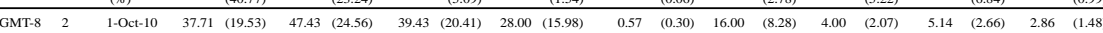
$\begin{array}{llllllllllllllllllllll}\text { GMT-8 } & 3 & 8 \text {-Oct-10 } & 43.43 & (19.44) & 42.86 & (19.18) & 20.00 & (8.95) & 10.86 & (5.88) & 2.29 & (1.02) & 23.43 & (10.49) & 29.14 & (13.04) & 10.29 & (4.60) & 2.29 & (1.02\end{array}$

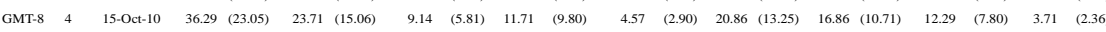
$\begin{array}{lllllllllllllllllllll}\text { GMT-8 } & 5 & 22-\text { Oct-10 } & 29.14 & (15.69) & 15.43 & (8.31) & 17.14 & (9.23) & 58.29 & (34.77) & 5.71 & (3.08) & 20.57 & (11.08) & 16.57 & (8.92) & 8.00 & (4.31) & 6.29 & (3.38\end{array}$

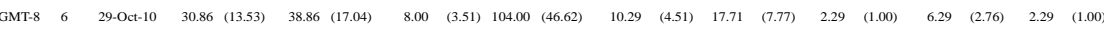

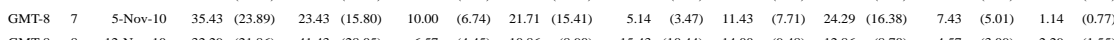
$\begin{array}{lllllllllllllllllllll}\text { GMT-8 } & 8 & 12-\mathrm{Nov}-10 & 32.29 & (21.86) & 41.43 & (28.05) & 6.57 & (4.45) & 10.86 & (8.90) & 15.43 & (10.44) & 14.00 & (9.48) & 12.86 & (8.70) & 4.57 & (3.09) & 2.29 & (1.55)\end{array}$ $\begin{array}{llllllllllllllllllll}\text { GMT-8 } & 9 & 19-\mathrm{Nov}-10 & 40.57(14.40) & 59.43 & (21.10) & 16.00 & (5.68) & 45.71 & (16.63) & 60.00 & (21.30) & 25.71 & (9.13) & 21.14 & (7.51) & 5.14 & (1.83) & 1.14 & (0.41)\end{array}$

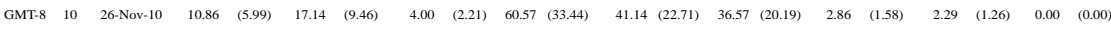
$\begin{array}{lllllllllllllllllllll}\text { GMT-8 } & 11 & \text { 3-Dec-10 } & 4.57 & (4.10) & 24.57 & (22.05) & 0.86 & (0.77) & 12.29 & (11.28) & 29.14 & (26.15) & 24.86 & (22.31) & 8.86 & (7.95) & 2.29 & (2.05) & 0.29 & (0.26)\end{array}$

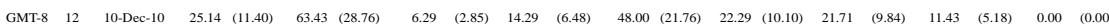
$\begin{array}{lllllllllllllllllllll}\text { GMT-8 } & 13 & \text { 17-Dec-10 } & 18.86 & (3.92) & 66.29 & (13.79) & 6.29 & (1.31) & 14.86 & (3.45) & 168.57 & (35.08) & 35.43 & (7.37) & 10.29 & (2.14) & 24.57 & (5.11) & 1.71 & (0.36\end{array}$

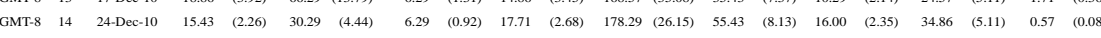

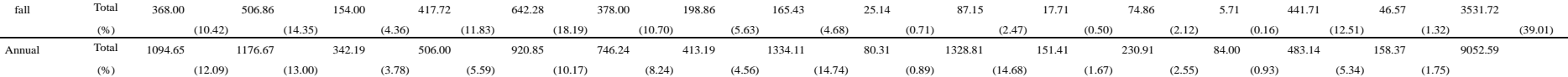

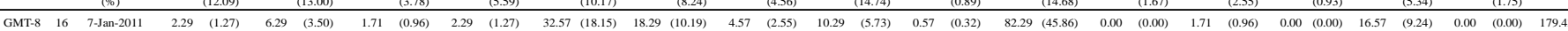

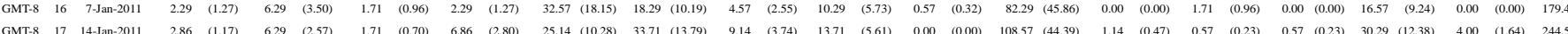
$\begin{array}{llllllllllllllllllllllllllllllllll}\text { GMT-8 } & 17 & 14-J a n-2011 & 2.86 & (1.17) & 6.29 & (2.57) & 1.71 & (0.70) & 6.86 & (2.80) & 25.14 & (10.28) & 33.71 & (13.79) & 9.14 & (3.74) & 13.71 & (5.61) & 0.00 & (0.00) & 108.57 & (44.39) & 1.14 & (0.47) & 0.57 & (0.23) & 0.57 & (0.23) & 30.29 & (12.38) & 4.00 & (1.64) & 244.57 \\ \text { GMT-8 } & 18 & 19-J a n-2011 & 0.00 & (0.00) & 1.14 & (0.66) & 0.00 & 0.00 & 1.14 & (0.66) & 6.86 & (3.95) & 2.86 & (1.64) & 1.71 & (0.99) & 2.86 & (1.64) & 0.00 & (0.00) & 154.86 & (89.14) & 0.00 & (0.00) & 0.00 & (0.00) & 0.00 & (0.00) & 2.29 & (1.32) & 0.00 & (0.00) & 173.71\end{array}$

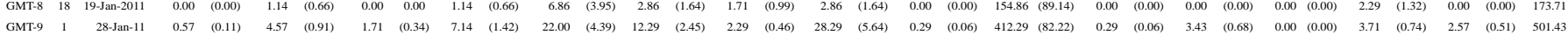

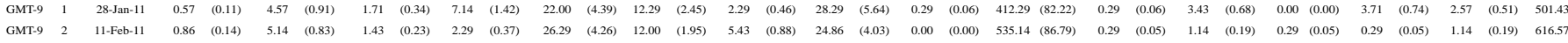
$\begin{array}{lllllllllllllllllllllllllllllllllllll}\text { GMT-9 } & 3 & 25-\mathrm{Feb}-11 & 9.71 & (2.45) & 20.57 & (5.19) & 9.71 & (2.45) & 8.00 & (2.02) & 82.86 & (20.91) & 43.71 & (11.03) & 22.57 & (5.70) & 79.14 & (19.97) & 1.14 & (0.29) & 108.57 & (27.40) & 0.00 & (0.00) & 7.14 & (1.80) & 1.14 & (0.29) & 0.29 & (0.07) & 2.00 & (0.50) & 396.29\end{array}$

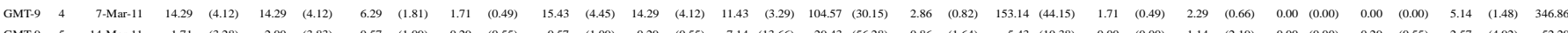
$\begin{array}{llllllllllllllllllllllllllllllllll}\text { GMT-9 } & 5 & 14-\mathrm{Mar}-11 & 1.71 & (3.28) & 2.00 & (3.83) & 0.57 & (1.09) & 0.29 & (0.55) & 0.57 & (1.09) & 0.29 & (0.55) & 7.14 & (13.66) & 29.43 & (56.28) & 0.86 & (1.64) & 5.43 & (10.38) & 0.00 & (0.00) & 1.14 & (2.19) & 0.00 & (0.00) & 0.29 & (0.55) & 2.57 & (4.92) & 52.29\end{array}$

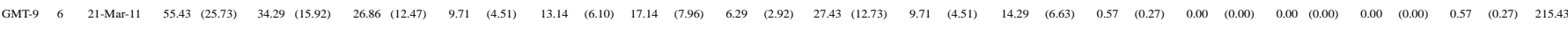

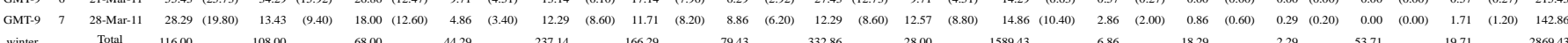

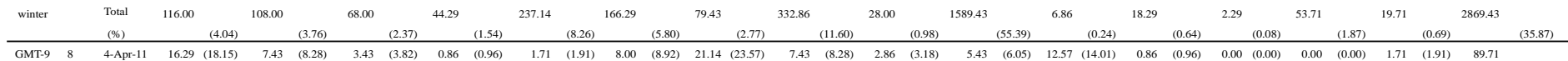
$\begin{array}{llrlllllllllllllllllll}\text { GMT-9 } & 8 & \text { 4-Apr-11 } & 16.29 & (18.15) & 7.43 & (8.28) & 3.43 & (3.82) & 0.86 & (0.96) & 1.71 & (1.91) & 8.00 & (8.92) & 21.14 & (23.57) & 7.43 & (8.28) & 2.86 & (3.18) \\ \text { GMT-9 } & 9 & 11-\text { Apr-11 } & 30.57 & (24.26) & 29.14 & (23.13) & 11.43 & (9.07) & 1.14 & (0.91) & 4.29 & (3.40) & 11.14 & (8.84) & 8.86 & (7.03) & 7.14 & (5.67) & 3.71 & (2.95)\end{array}$

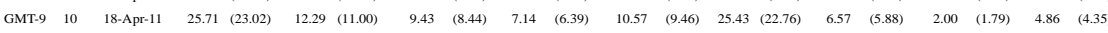

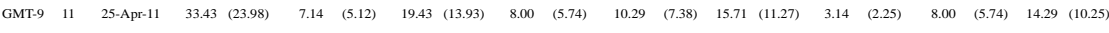
$\begin{array}{lllllllllllllllllllll}\text { GMT-9 } & 12 & 5 / 2 / 2011^{*} & 10.00 & (20.83) & 2.57 & (5.36) & 5.43 & (11.31) & 1.14 & (2.38) & 1.43 & (2.98) & 7.71 & (16.07) & 4.86 & (10.12) & 4.29 & (8.93) & 4.57 & (9.52)\end{array}$

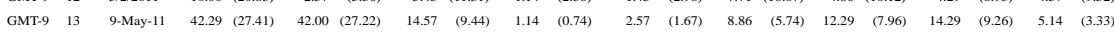

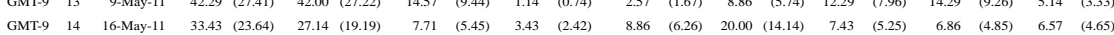

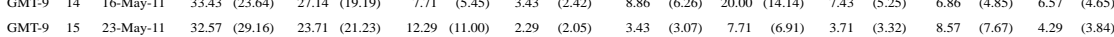

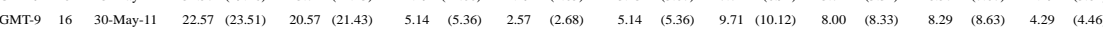
$\begin{array}{lllllllllllllllllllll}\text { GMT-9 } & 17 & 6 \text {-Jun-11 } & 35.43 & (20.39) & 40.57 & (23.36) & 7.43 & (4.28) & 0.57 & (0.33) & 2.29 & (1.32) & 5.14 & (2.96) & 20.57 & (11.84) & 37.71 & (21.71) & 7.43 & (4.28\end{array}$

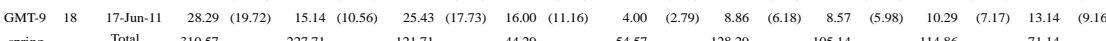
$\begin{array}{lllllllllllll}4.86 & (3.85) & 8.57 & (6.80) & 2.00 & (1.59) & 0.86 & (0.68) & 0.00 & (0.00) & 2.29 & (1.81) & 126.00 \\ 3.14 & (2.81) & 2.29 & (2.05) & 1.14 & (1.02) & 0.00 & (0.00) & 0.00 & (0.00) & 1.14 & (1.02) & 111.71\end{array}$

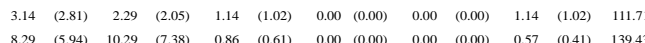

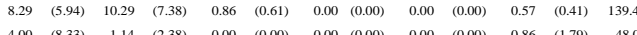
$\begin{array}{lllllllllllll}4.00 & (8.33) & 1.14 & (2.38) & 0.00 & (0.00) & 0.00 & (0.00) & 0.00 & (0.00) & 0.86 & (1.79) & 48.00 \\ 2.57 & (1.67) & 0.57 & (0.37) & 4.29 & (2.78) & 0.00 & (0.00) & 0.00 & (0.00) & 4.00 & (2.59) & 154.29\end{array}$ $\begin{array}{lllllllllllll}2.57 & (1.67) & 0.57 & (0.37) & 4.29 & (2.78) & 0.00 & (0.00) & 0.00 & (0.00) & 4.00 & (2.59) & 154.29 \\ 8.86 & (6.26) & 4.57 & (3.23) & 3.71 & (2.63) & 0.00 & (0.00) & 0.00 & (0.00) & 2.86 & (2.02) & 141.43\end{array}$ $\begin{array}{lllllllllllll}2.57 & (2.30) & 1.71 & (1.53) & 1.71 & (1.53) & 0.00 & (0.00) & 0.00 & (0.00) & 7.14 & (6.39) & 111.71\end{array}$ $\begin{array}{lllllllllllll}1.71 & (1.79) & 1.43 & (1.49) & 1.71 & (1.79) & 0.29 & (0.30) & 0.00 & (0.00) & 4.57 & (4.76) & 96.00\end{array}$ $\begin{array}{lllllllllllll}1.71 & (0.99) & 2.29 & (1.32) & 7.43 & (4.28) & 0.00 & (0.00) & 0.00 & (0.00) & 5.14 & (2.96) & 173.7\end{array}$ $\begin{array}{lllllllllllll}5.71 & (3.98) & 4.00 & (2.79) & 1.43 & (1.00) & 0.00 & (0.00) & 0.00 & (0.00) & 2.57 & (1.79) & 143.4\end{array}$

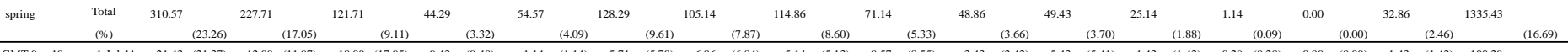

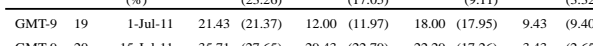

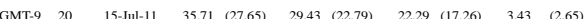
$\begin{array}{llllllllllllllllllllll}\text { GMT-9 } & 21 & 31-\text { Jul-11 } & 22.89 & (31.60) & 7.78 & (10.74) & 12.44 & (17.18) & 1.33 & (1.84) & 1.33 & (1.84) & 4.22 & (5.83) & 7.11 & (9.82) & 3.33 & (4.60) & 5.33 & (7.36)\end{array}$ $\begin{array}{llllllllllllllllllll}\text { GMT-10 } & 1 & \text { 21-Aug-11 } & 30.43 & (30.74) & 22.57(22.80) & 11.00 & (11.11) & 3.43 & (3.46) & 0.14 & (0.14) & 1.00 & (1.01) & 6.43 & (6.49) & 5.57 & (5.63) & 3.43 & (3.46\end{array}$ $\begin{array}{llllllllllllllllllll}\text { GMT-10 } & 2 & 4-S e p-11 & 52.86(31.41) & 42.00 & (24.96) & 36.57 & (21.73) & 0.00 & (0.00) & 0.00 & (0.00) & 0.29 & (0.17) & 20.86 & (12.39) & 5.14 & (3.06) & 3.14 & (1.87\end{array}$

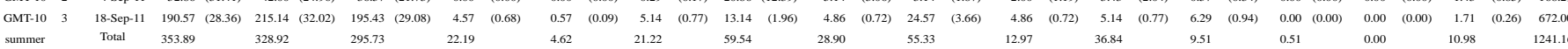
$\begin{array}{lllllllllllll}3.43 & (3.42) & 5.43 & (5.41) & 1.43 & (1.42) & 0.29 & (0.28) & 0.00 & (0.00) & 1.43 & (1.42) & 100.29 \\ 1.14 & (0.88) & 8.29 & (6.42) & 0.86 & (0.66) & 0.00 & (0.00) & 0.00 & (0.00) & 1.43 & (1.11) & 129.14\end{array}$ $\begin{array}{rrrrrrrrrrrrr}1.14 & (0.88) & 8.29 & (6.42) & 0.86 & (0.66) & 0.00 & (0.00) & 0.00 & (0.00) & 1.43 & (1.11) & 129.14 \\ 1.11 & (1.53) & 3.56 & (4.91) & 0.22 & (0.31) & 0.22 & (0.31) & 0.00 & (0.00) & 1.56 & (2.15) & 72.44\end{array}$

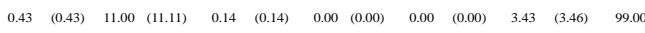

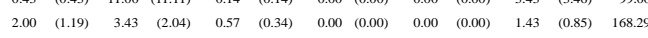

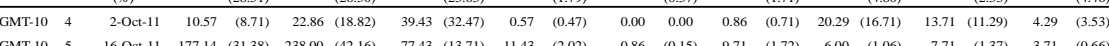

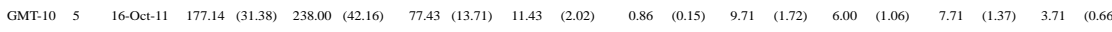
$\begin{array}{llllllllllllllllllllll}\text { GMT-10 } & 6 & 26-O c t-11 & 46.86 & (24.85) & 13.71 & (7.27) & 95.43 & (50.61) & 2.86 & (1.52) & 1.14 & (0.61) & 5.14 & (2.73) & 9.14 & (4.85) & 6.29 & (3.33) & 1.14 & (0.61)\end{array}$

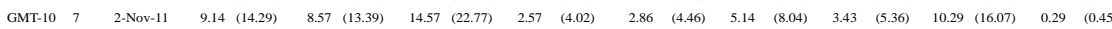
$\begin{array}{lllllllllllllllllllll}\text { GMT-10 } & 8 & 9 \text {-Nov-11 } & 10.57 & (9.76) & 10.00 & (9.23) & 8.29 & (7.65) & 13.43 & (12.40) & 24.57 & (22.69) & 18.86 & (17.41) & 3.14 & (2.90) & 9.14 & (8.44) & 2.86 & (2.64\end{array}$

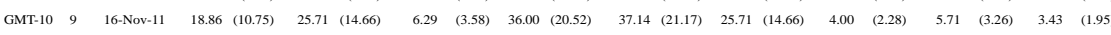

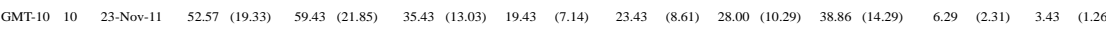
$\begin{array}{llllllllllllllllllllll}\text { GMT-10 } & 11 & 30-\mathrm{Nov}-11 & 9.14 & (14.81) & 7.43 & (12.04) & 11.71 & (18.98) & 2.57 & (4.17) & 7.71 & (12.50) & 6.29 & (10.19) & 9.14 & (14.81) & 2.29 & (3.70) & 2.86 & (4.63)\end{array}$ $\begin{array}{lllllllllllllllllllll}\text { GMT-10 } & 12 & 7 \text {-Dec-11 } & 26.29 & (10.31) & 41.71 & (16.37) & 33.14 & (13.00) & 22.29 & (8.74) & 24.57 & (9.64) & 62.86 & (24.66) & 16.57 & (6.50) & 13.14 & (5.16) & 2.29 & (0.90)\end{array}$

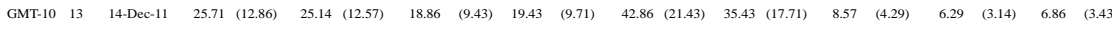
$\begin{array}{lllllllllllllllllllll}\text { GMT-10 } & 14 & 21-\text { Dec-11 } & 14.29 & (4.43) & 28.57 & (8.87) & 33.71 & (10.46) & 9.71 & (3.01) & 28.00 & (8.69) & 25.71 & (7.98) & 39.43 & (12.23) & 18.86 & (5.85) & 11.43 & (3.55)\end{array}$

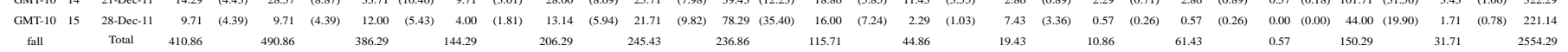

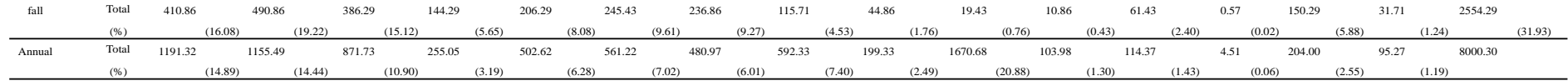
$\begin{array}{rrrrrrrrrrrrr}1.14 & (0.94) & 1.71 & (1.41) & 3.71 & (3.06) & 0.00 & (0.00) & 0.00 & (0.00) & 2.29 & (1.88) & 121.4 \\ 2.00 & (0.35) & 2.29 & (0.40) & 25.14 & (4.45) & 0.00 & (0.00) & 0.00 & (0.00) & 3.43 & (0.61) & 564.57\end{array}$ $\begin{array}{lllllllllllll}2.00 & (0.00) & 0.57 & (0.30) & 5.14 & (2.73) & 0.00 & (0.00) & 0.00 & (0.00) & 1.14 & (0.61) & 188.57\end{array}$ $\begin{array}{lllllllllllll}4.29 & (6.70) & 0.57 & (0.89) & 1.43 & (2.23) & 0.00 & (0.00) & 0.00 & (0.00) & 0.86 & (1.34) & 64.00\end{array}$ $\begin{array}{lllllllllllll}0.57 & (0.53) & 0.57 & (0.53) & 1.43 & (1.32) & 0.00 & (0.00) & 0.00 & (0.00) & 4.86 & (4.49) & 108.29\end{array}$ $\begin{array}{lllllllllllll}0.00 & (0.00) & 1.14 & (0.65) & 6.29 & (3.58) & 0.00 & (0.00) & 0.00 & (0.00) & 5.14 & (2.93) & 175.43 \\ 0.00 & (0.00) & 0.00 & (0.00) & 4.00 & (1.47) & 0.00 & (0.00) & 0.00 & (0.00) & 1.14 & (0.42) & 272.00\end{array}$ $\begin{array}{lllllllllllll}0.00 & (0.00) & 0.00 & (0.00) & 1.14 & (1.85) & 0.00 & (0.00) & 0.00 & (0.00) & 1.43 & (2.31) & 61.71\end{array}$ $\begin{array}{llllllllllllll}0.00 & (0.00) & 0.00 & (0.00) & 6.86 & (2.69) & 0.00 & (0.00) & 0.57 & (0.22) & 4.57 & (1.79) & 254.86\end{array}$

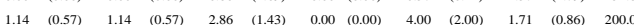

